\title{
A Prioritized Network Coding Scheme based E-WP Algorithm for Packet Recovery in DVB-IPDC System
}

\author{
Lian Wang ${ }^{1,2}$ and Jiehao Wang ${ }^{1}$ \\ 1. School of Computer Science and Technology, Chongqing University of Posts \\ and Telecommunications, Chongqing 400065, P. R. China \\ 2. Provincial Key Laboratory of Information Coding and Transmission, School \\ of Information Science and Technology, Southwest Jiaotong University, \\ Chengdu 610031, P. R. China \\ Email:wanglian@cqupt.edu.cn,wjh123987@hotmail.com
}

\begin{abstract}
DVB-H is developed to broadcast digital videos to mobile handsets, but data loss is a concern due to the wireless broadcasting nature. In this paper, take WiMAX as an IPbased wireless network to recover the loss packets in DVB-H, and take E-WP based on network coding as the encoding packet selection algorithm. Furthermore, a prioritized scheme EWP-PNC based on E-WP packet selection algorithm is proposed. In this scheme, take a base station in WiMAX as encoding node to encode any two packets that meet the encoding and decoding necessary and sufficient condition into an encoding recovery packet according to the current lost packet distribution, and calculate the benefit of each encoding packet to decide the final priority of all encoding packets. The goals of this scheme are to improve the lost packet recovery ratio and reduce the discarded packet ratio. According to the simulation results, the validity of this scheme is proved.
\end{abstract}

Keywords: network coding, DVB-IPDC, complete-decoding, wireless network

\section{Introduction}

DVB-H (Digital Video Broadcasting - Handheld), the specification for bringing broadcast services to battery-powered Mobile Handsets (MHs), was formally adopted as an ETSI standard in November 2004 [1]. However, due to the wireless channel characteristic and wireless broadcast properties, MHs are vulnerable to lose packets in a wireless environment $[2,3]$. Some studies are trying to deal with the DVB-H data loss problem, [4] adopts a cellular network to transmit repair data, [5] proposes an FEC scheme to deliver DVB-H lost packets to improve transmission efficiency, and [6] integrates DVB-H with a $3 \mathrm{G}$ network to transmit data for the recovery purpose. However, none of the above studies take the correlation among different lost packets into consideration. IP datacast over DVB-H (DVB-IPDC) suggests a separate IP-based wireless network as recovery channel. MHs interact with the system, MHs can submit requests to the BS [7][8], after the BS receives the requests from all MHs, BS as an encoding node encode any two packets that meet the encoding and decoding necessary and sufficient condition into an encoding recovery packet, then broadcast the encoding packet to MHs.

Now, network coding is widely adopted to deal with the loss packets in wireless network due to wireless broadcast characteristics. The current typical algorithms of network coding used in wireless network retransmission mainly include Random-Pick [9], Most-Least [10], Hamming-Distance [11], In Random Pick, encoding node randomly selects two packets from all packets that need to be retransmitted or packets available, and performs XOR operation on the selected packets to generate encoding packet for 
retransmission. Although Random Pick is simple to implement, but the high retransmission efficiency cannot be achieved and only better than ARQ [12]. In MostLeast, the base station selects the two packets with the most retransmission requests and the least retransmission requests in generating an encoded packet for retransmission [13]. In Hamming-Distance, the base station selects the two packets with the largest hamming distance from the packet distribution matrix for encoding [14]. In this paper, an adaptive priority packet recovery scheme based on E-WP (Extended Weight Pick Algorithm) is proposed, and $E-W P$ is the packet selection algorithm based on network coding is employed. While due to the priority or the timing constraint of different lost packets, such as the real-time packets should be differentiated from non-real-time packets by giving a higher priority[15,16], this scheme calculate the weighted values, and then the base station will calculate the benefit to broadcast the encoding packet.

This paper is organized as follows. Section 1 is introduction. Section 2 defines the network model. Section 3 is performance analysis. Section 4 is EWP-PNC scheme description. Section 5 is performance evaluation.

\section{Network Model}

To support DVB-IPDC, WiMAX networks is adopted as an example to serve as the datacast channel[18,19]. As shown in Fig.1, that the WiMAX network is used as the recovery channel in DVB-IPDC[17][18] Suppose that the WiMAX cell has $M(M>2)$ receivers $\mathrm{R}_{k}(1 \leq k \leq M)$ During each frame, the MHs submit their requests of lost packets in Tsub period, while in Trec, The BS broadcasts encoding packets to MHs for recovering lost packets. In addition, each encoding packet has a deadline, if exceed the deadline the lost packets still have not been recovered, they will be discarded.

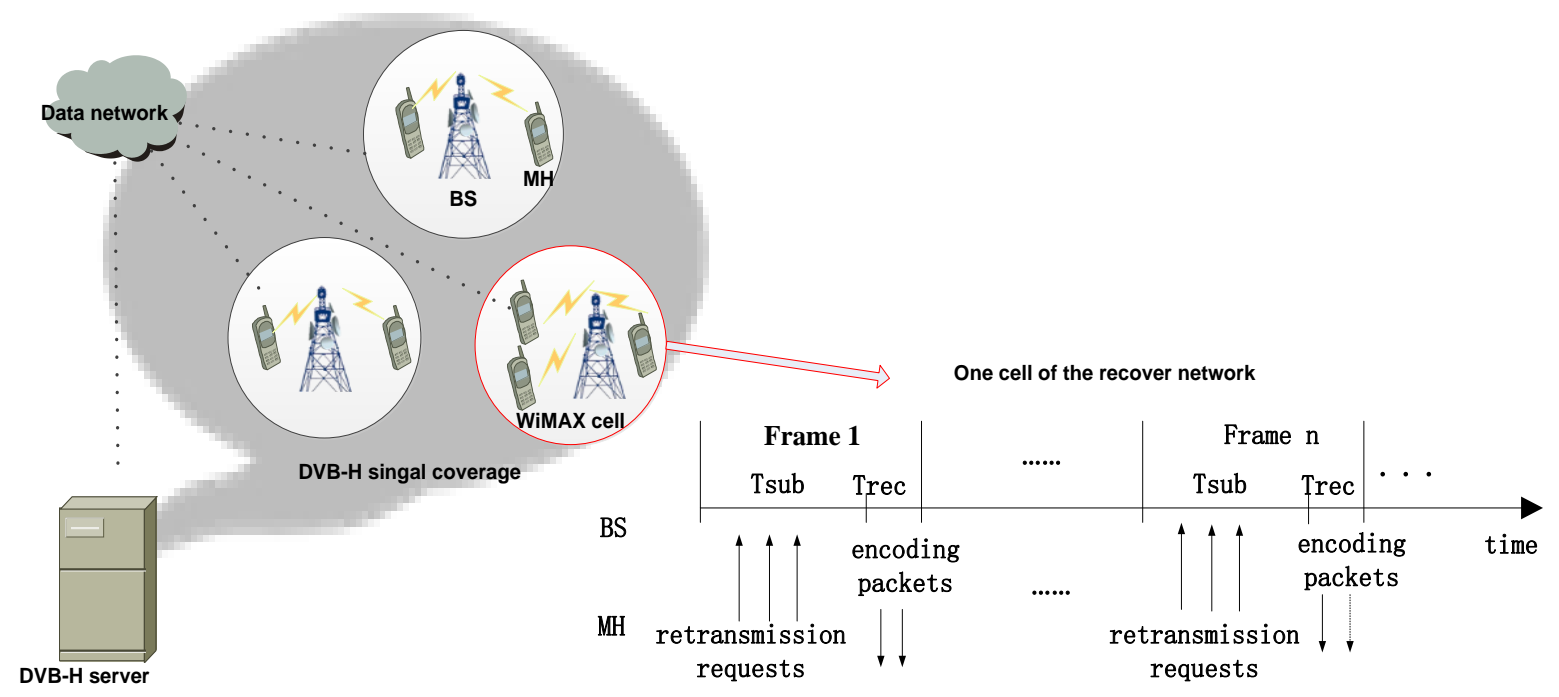

Figure 1. Network Model

\section{Performance Analysis}

In this section, the retransmission performance has been compared between the scheme based on network coding and the traditional scheme without network coding by mathematical analysis. The comparison parameter is the average number of retransmission to receive a packet successfully. Suppose LA represents the average number of retransmission without network coding, and LB represents the average number 
of retransmission based on network coding. Two lemmas have been defined as follows [19].

Lemma 1: When the numbers of receiver is $M(M>2)$, the average number of retransmission without network coding is:

$$
L A=\sum_{i_{1}, i_{2}, \ldots i_{M}} \frac{(-1)^{i_{1}+i_{2} \ldots+i_{M-1}}}{1-p_{1}^{i_{1}} p_{2}^{i_{2}} \ldots p_{M}^{i_{M}}}
$$

Where $i_{1}, i_{2} \ldots, i_{n} \in\{0,1\}$, and $\exists i_{j} \neq 0$.

Lemma 2: When the numbers of receiver is $M(M>2)$, the average number of retransmission based on network coding is:

$$
L B=\frac{1}{1-\max _{i \in\{1,2, \ldots M\}}\left\{p_{i}\right\}}
$$

The encoding gain ratio between these two schemes is defined as:

$$
\text { When } p_{i}=p(i=1,2, \ldots M)
$$

$$
G=\frac{\sum_{i_{1}, i_{2}, \ldots i_{M}} \frac{(-1)^{i_{1}+i_{2} \ldots+i_{M-1}}}{1-p_{1}^{i_{1}} p_{2}^{i_{2}} \ldots p_{M}^{i_{M}}}}{\frac{1}{1-\max _{i \in\{1,2, \ldots M\}}\left\{p_{i}\right\}}}=\frac{\sum_{K=1}^{M} \frac{(-1)^{K-1}\left(\begin{array}{c}
M \\
K
\end{array}\right)}{1-p^{K}}}{\frac{1}{1-p}}
$$

As shown in the above, when $M$ is bigger, the encoding gain $G$ is bigger, so the retransmission scheme based on network coding is superior to the retransmission scheme without network coding.

\section{EWP-PNC Scheme}

EWP-PNC is a prioritized scheme based Weight Pick algorithm for packet recovery, which takes two aspects into consideration, maximize the total number of packets recovered on each $\mathrm{MH}$ while minimize the aggregate number of packet discarded due to out of deadline. How to reach these purposes, first the efficient recovery packets selection algorithm is introduced, second the adaptive priority recover scheme highlights the delay constrain of required packets.

\subsection{Relative Definitions}

Definition 1: Packet Distribution Matrix (PDM) has $\mathrm{M}$ columns and $\mathrm{N}$ rows, in which each column vector $P_{i}(1 \leq i \leq M)$ stands for the packet $i$, while each row vector $R_{j}(1 \leq j \leq N)$ stands for receiver $\mathrm{j}$. 
Definition 2: Complete Decoding means all the lost packets can be decoded to the specified receiver. Assume the lost packets vector is $\rho\left(P_{1}, \cdots \rho\left(P_{M}\right)\right)$ to packet $P_{i}(1 \leq i \leq M)$, the specified packet can be complete decoded if and only if there is no more than one ' 0 ' at each row of the matrix $F=\left[\rho\left(P_{1}^{T}\right), \cdots \rho\left(P_{M}{ }^{T}\right)\right]$.

Definition 3: The upper bound of retransmission is the maximum of retransmission times. In this scheme the upper bound of retransmission is no more than the total number of requested packets.

\subsection{The Retransmission Packet Selection Algorithm}

The packet selection algorithm of this paper, named E-WP is proposed, which combines the major idea of Weight Pick [20]. Especially because the lost packets in DVB-IPDC with high spatial or temporal correlation, and the feasibility of the algorithm in the real application, E-WP is simplified compared with the original Weight Pick algorithm: first the number of combination packets is fixed, the upper bound is 2 . Second under the premise of decoding condition, give the priority to the required packet with maximum-weight. Third, the number of retransmission is less than $N q$ the number of lost packets in the worst case. E-WP can be looked as a special case of Weight Pick. $N q$ is the total number of lost packets of all MHs.

The main steps of the EWP-PNC algorithm are summarized as follow:

Step1: If the number of original packet is less than 2, directly encode the packets or retransmit the original individually, otherwise go to Step 2.

Step2: Search for two columns in the PSM which can complete decoding. If the searching result is not null, then combine the two columns to an encoding packet. And the rest columns which cannot complete decoding retransmit the requested packet individually. If the searching result is null, retransmit these requested packets individually.

Step3: According to the weighted values, calculate the benefit of the above encoding packets or individual packets, and then based on the size of benefit of these packets, retransmit the encoding packet with the maximum-weight to the MHs. If one packet has been received successfully by all the MHs, each element of the corresponding column is 1 , and the column will be deleted from the PSM, then the PSM is updated. Go back to step1.

Step4: If all the lost packets have been recovered by all the receivers, the retransmission procedure is finished.

\subsection{EWP-PNC}

EWP-PNC scheme adopts the packet selected algorithm based on E-WP in order to improve the retransmission efficiency. The corresponding weighted values of each encoding packet are calculated. $W\left(P_{i}, C_{j}\right)$ is weighted value which reflects the priority of the coding packet, the value is bigger that the coding retransmission packet has higher retransmission probability to be recovered, where $P_{i}$ is the original packets, and $C_{j}$ is coding packet. $W\left(P_{i}, C_{j}\right)$ takes two factors into consideration the packets recovery and the packet emergency. Where $\lambda$ and $\mu$ are the scaling factors that meet $(\lambda+\mu=1)$. According to the application background, adjust the two factors value.

$$
W\left(P_{i}, C_{j}\right)=\lambda \times \frac{\sum E W P\left(P_{i}, C_{j}\right)}{N_{q}}+\mu \times\left(\frac{1}{\min \left\{L F\left(R_{K}\right)\right\}-t_{c}}\right)
$$


Where $\min \left\{D\left(R_{K}\right)\right\}$ means the earliest deadline of requested packets which can be recovered by the encoding packet $C_{j}, t_{c}$ is the current time. Where $\sum E W P\left(P_{i}, C_{j}\right)$ equals to the total number of original $P_{i}$ decoded from the coding packet $C_{j}$ at individual $\mathrm{MH}, N_{q}$ is the total number of lost packets of all $\mathrm{MHs}, \mathrm{M}$ is the total number of receiver. According to (4), each $W\left(P_{i}, C_{j}\right)$ can be achieved, then $W\left(C_{j}\right)$ the weighted value of $C_{j}$ equals to the sum of all weighted value $W\left(P_{i}, C_{j}\right)$ that encoding packet $C_{j}$ can decode out $P_{i}$.

The BS can dynamically assign specified deadline $\operatorname{LF}\left(R_{k}\right)$ to each receiver rather than fixed time during retransmission stage according to its emergence. Namely the retransmission packet only has the deadline $\operatorname{LF}\left(R_{k}\right)$ transmission opportunities, otherwise the packet arrives too late to be used. The basic idea of EWP-PNC is further explained by the following example. As shown in Table.1, ' 1 'means that the packet is received successfully by the MH in PSM, or else failed. As shown in Table2, the received and required packets are listed, where $\operatorname{LF}\left(R_{k}\right)$ is deadline of each packet to the corresponding $\mathrm{MH}$. That means the requested packets should be reached before deadline $\operatorname{LF}\left(R_{k}\right)$.According to the packet selection algorithm the final encoding packets are $C_{1}=P_{1} \oplus P_{3}, C_{2}=P_{1} \oplus P_{4}, C_{3}=P_{3} \oplus P_{4}, C_{4}=P_{2}$.

\section{Table 1.Packet Status Matrix (PSM) Table 2.Received and Required Packets List}

\begin{tabular}{|c|c|c|c|c|}
\hline & $\mathrm{P}_{1}$ & $\mathrm{P}_{2}$ & $\mathrm{P}_{3}$ & $\mathrm{P}_{4}$ \\
\hline $\mathrm{R}_{1}$ & 0 & 1 & 1 & 1 \\
\hline $\mathrm{R}_{2}$ & 1 & 0 & 0 & 1 \\
\hline $\mathrm{R}_{3}$ & 0 & 0 & 1 & 1 \\
\hline $\mathrm{R}_{4}$ & 1 & 1 & 1 & 0 \\
\hline $\mathrm{R}_{5}$ & 1 & 0 & 1 & 0 \\
\hline
\end{tabular}

\begin{tabular}{|c|c|c|c|}
\hline RREQ & $\mathrm{Si}$ & $\mathrm{Qi}$ & $\mathrm{LF}\left(R_{k}\right)$ \\
\hline $\mathrm{R} 1$ & $\{\mathrm{P} 2, \mathrm{P} 3, \mathrm{P} 4\}$ & $\{\mathrm{P} 1\}$ & 9 \\
\hline $\mathrm{R} 2$ & $\{\mathrm{P} 1, \mathrm{P} 4\}$ & $\{\mathrm{P} 2, \mathrm{P} 3\}$ & 11 \\
\hline $\mathrm{R} 3$ & $\{\mathrm{P} 3, \mathrm{P} 4\}$ & $\{\mathrm{P} 1, \mathrm{P} 2\}$ & 7 \\
\hline $\mathrm{R} 4$ & $\{\mathrm{P} 1, \mathrm{P} 2, \mathrm{P} 3\}$ & $\{\mathrm{P} 4\}$ & 8 \\
\hline R5 & $\{\mathrm{P} 1, \mathrm{P} 3\}$ & $\{\mathrm{P} 2, \mathrm{P} 4\}$ & 10 \\
\hline
\end{tabular}

The details are as follows. First, search for the PSM1, find $\left\{\mathrm{P}_{1}, \mathrm{P}_{3}\right\},\left\{\mathrm{P}_{1}, \mathrm{P}_{4}\right\},\left\{\mathrm{P}_{3}, \mathrm{P}_{4}\right\}$ can complete decoding, then combine them to an encoding packet $P_{1} \oplus P_{3} 、 P_{1} \oplus P_{4}$ 、 $P_{3} \oplus P_{4}$. Second, the rest column $\left\{\mathrm{P}_{2}\right\}$ cannot complete decoding, set it as an individual packet for retransmitted. Third, suppose $\lambda=\mu=0.5, t_{c}=3$ the priority weighted values are calculated as following:

$$
\begin{aligned}
& w\left(P_{1}, C_{1}\right)=\lambda \times \frac{\sum E W P\left(P_{1}, C_{1}\right)}{N_{q}}+\mu \times \frac{1}{\min \left\{D\left(R_{1}\right), D\left(R_{3}\right)\right\}-3}=0.5 \times \frac{2}{8}+0.5 \times \frac{1}{7-3}=0.25 \\
& w\left(P_{3}, C_{1}\right)=\lambda \times \frac{\sum E W P\left(P_{3}, C_{1}\right)}{N_{q}}+\mu \times \frac{1}{\min \left\{\left(D\left(R_{2}\right)\right\}-3\right.}=0.5 \times \frac{1}{8}+0.5 \times \frac{1}{11-3}=0.125
\end{aligned}
$$

And then $W\left(C_{1}\right)=0.375, W\left(C_{2}\right)=0.475$ 


$$
W\left(C_{3}\right)=w\left(P_{3}, C_{3}\right)+w\left(P_{4}, C_{3}\right)=0.35, W\left(C_{4}\right)=w\left(P_{2}, C_{4}\right)=0.3125
$$

From the final calculation result $W\left(C_{2}\right)>W\left(C_{1}\right)>W\left(C_{3}\right)>W\left(C_{4}\right)$, the priority sequence of encoding packet is $C_{2}>C_{1}>C_{3}>C_{4}$. In this case, $C_{2}$ first select since it has the maximum weight and thus retransmit $P_{1} \oplus P_{4}$ to MHs, when the MHs recover the lost packets successfully by encoding packet $P_{1} \oplus P_{4}$, the corresponding column $\left\{\mathrm{P}_{1}, \mathrm{P}_{4}\right\}$ will be undated as, and then delete them from PSM, update PSM. As shown in Table 3, Search for the PSM1, there are no complete decoding effective columns in PDM1. So retransmit $\mathrm{P}_{2} 、 \mathrm{P}_{3}$ individually.

\section{Table 3. Packet Status Matrix (PSM1)}

\begin{tabular}{|c|c|c|}
\hline & $\mathrm{P}_{2}$ & $\mathrm{P}_{3}$ \\
\hline $\mathrm{R}_{1}$ & 1 & 1 \\
\hline $\mathrm{R}_{2}$ & 0 & 0 \\
\hline $\mathrm{R}_{3}$ & 0 & 1 \\
\hline $\mathrm{R}_{4}$ & 1 & 1 \\
\hline $\mathrm{R}_{5}$ & 0 & 1 \\
\hline
\end{tabular}

$W\left(P_{2}, C_{5}\right)=\lambda \times \frac{\sum E W P\left(P_{2}, C_{5}\right)}{N_{q}}+\mu \times \frac{1}{\min \left\{L F\left(R_{2}\right), L F\left(R_{4}\right), L F\left(R_{5}\right)\right\}-3}=0.5 \times \frac{2}{4}+0.5 \times \frac{1}{7-3}=0.375$

$W\left(P_{3}, C_{6}\right)=\lambda \times \frac{\sum E W P\left(P_{3}, C_{6}\right)}{N_{q}}+\mu \times \frac{1}{\min \left\{L F\left(R_{2}\right)\right\}-3}=0.5 \times \frac{1}{4}+0.5 \times \frac{1}{11-3}=0.1875$

$W\left(C_{5}\right)=W\left(P_{2}, C_{5}\right)=0.375$

$W\left(C_{6}\right)=W\left(P_{3}, C_{6}\right)=0.1875$

According to the calculation result, the priority sequence of encoding packet is $C_{5}>C_{6}$, so retransmit $\mathrm{P}_{2}$ to the MHs firstly, then the corresponding column $\left\{\mathrm{P}_{2}\right\}$ will be undated as $\{1,1,1,1,1\}^{T}$, and then delete it from PSM1, update PSM1. Now, only packet $\mathrm{P}_{3}$ has not been recovered, retransmit $\mathrm{P}_{3}$ directly. The whole retransmission procedure is finished. The encoding packet transmitted is $P_{1} \oplus P_{4} 、 P_{2} 、 P_{3}$. Due to this scheme which can dynamic combination number in an encoding packet and dynamic updating packet distributing information, so only in 3 slots all the lost packets can be recovered.

\section{Performance Evaluation}

Assume the transmit rate is a constant rate and the error ratio of reception rate at each $\mathrm{MH}$ is $30 \%$ at every time slot. The ratios of discarded packets is defined as the aggregate number of packets discarded by the scheme over the aggregate number of queried packets by all MHs. Obviously, the lower ratio of discarded packets means the scheme has a better performance since it can alleviate the video data discard. Set Tsub= 10 slots and Trec $=5$ slots, and the packet deadline is $1,2,3,4$, and 5 time slots respectively, right after each Tsub period. The performance comparisons are between EWP-PNC and PNC (Prioritized Network Coding) [21]. 


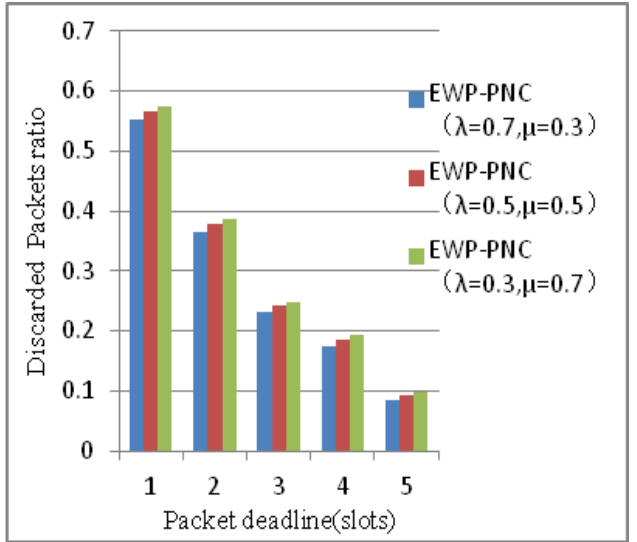

Figure 2.The Discarded Packets Ratio Comparison among Different Value of $\lambda$ and $\mu$ in EWP-PNC

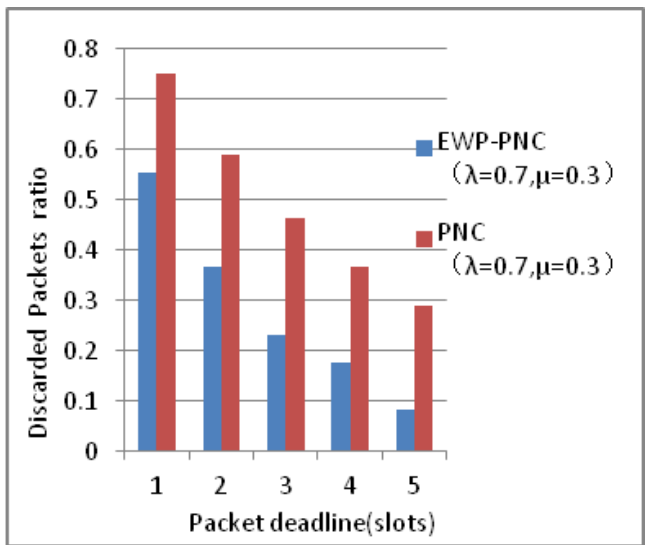

Figure 3.The Discarded Packets Ratio Comparison between EWP-

PNC and $\operatorname{PNC}^{(\lambda=0.7, \mu=0.3)}$

As shown in Figure 2, when the parameter $\lambda$ is increased, the discarded packets ratio of EWP-PNC is decreased obviously. Because $\lambda$ stands for the advantage of the retransmission packet selection algorithm which only take packet recovery into consideration。 While $\mu$ stands for the emergency of the lost packets for recovery. So the larget the $\lambda$ is, the more required packets can be recovered by MHs and also the discarded packets ratio is lower.

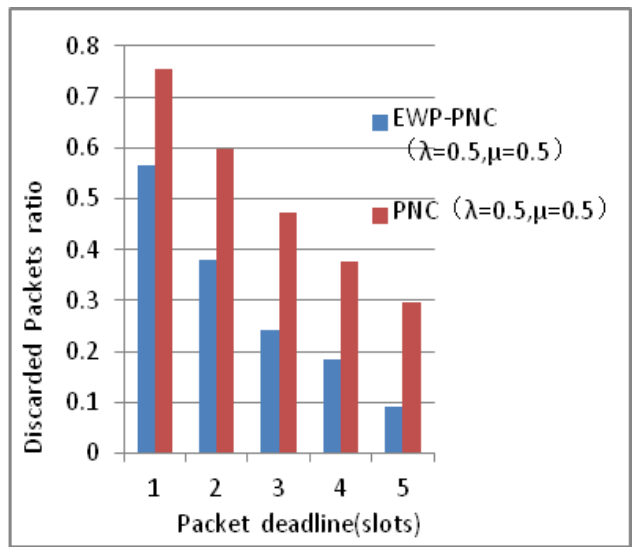

Figure 4. The Discarded Packets Ratio Comparison between EWP$\operatorname{PNC}$ and $\operatorname{PNC}^{(\lambda=0.5, \mu=0.5)}$

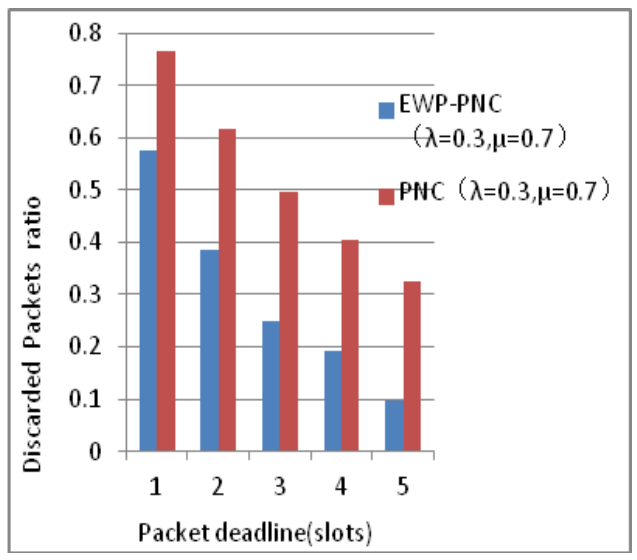

Figure 5.The Discarded Packets Ratio Comparison between EWP$\operatorname{PNC}$ and $\operatorname{PNC}^{(\lambda=0.3, \mu=0.7)}$

Figure.3, Figure 4 and Figure.5 show that, no matter how the value of $\lambda$ and $\mu$ change, the discarded packets ratio of HD-PNC always lower than PNC. This is because the packet selection algorithm of this paper is superior to PNC. The encoding algorithm E-HM dynamically determine the encoded packet and update PSM dynamically, in addition the priority scheme can equally treat with the packet recovery priority and time priority, furthermore according to the priority preference, adjust the factors to realize is feasible. 


\section{Conclusions}

DVB-H is developed to broadcast digital videos to MHs, but data loss is a concern due to the unreliable wireless. DVB-IPDC can integrate with an IP-based wireless network to complement the data loss problem in DVB-H. In this paper, a prioritized network coding scheme based E-WP algorithm for packet recovery in DVB-IPDC system (EWP-PNC) is proposed. E-WP algorithm can not only reduce the number of retransmission, but also can effectively reduce the number of discarded packets. According to the simulation result, the performance of EWP-PNC is superior to that of PNC .

\section{Acknowledgements}

This work is supported by the Program for Innovation Team Building at Institutions of Higher Education in Chongqing (Grant No. KJTD201310) and the National Basic Research Program of China (Grant No. 2012CB316100).

\section{Reeferences}

[1] G. Faria, J.A. Henriksson, E.Stare, and P.bTalmola, "DVB-H: digital broadcast services to handheld devices", Proceedings of the IEEE, (2006).

[2] O. Eerenberg, A Koppelaar, and AM. Stuivenwold, de With PHN. "IP-recovery in the DVB-H link layer for TV on mobile", Proc. IEEE International Conference on Consumer Electronic, (2011).

[3] W.H. Yang, Y.C. Wang, Y.C. Tseng, and B. S. P. Lin, "Spatial and temporal packet recovery schemes for DVB-H systems through IP-relay wireless networks", Communications, ICC '09. IEEE International Conference on, (2009).

[4] D. Gomez-Barquero, and A. Bria, "Repair mechanisms for broadcast transmissions in hybrid cellular \& DVBH systems", Proc. IEEE International Symposium on Wireless Communication Systems, (2006).

[5] D. Gomez-Barquero, D. Gozalvez and N. Cardona, "Application layer FEC for mobile TV delivery in IP datacast over DVB-H systems", IEEE Transactions on Broadcasting, vol.55, no.2 (2009), pp.396406.

[6] D. Gomez-Barquero, N. Cardona, A. Bria and J. Zander, "Affordable mobile TV services in hybrid cellular and DVB-H systems", IEEE Network, vol.21, no.2, (2007), pp.34-40.

[7] ETSI TR 102 469, "Digital video broadcasting (DVB)", IP datacast over DVB-H: architecture. European Telecommunications Stands Institute, (2006).

[8] M. Kornfeld, and G. May, "DVB-H and IP Datacast-broadcast to handheld devices", IEEE Transactions on Broadcasting, vol.53, no.1, (2007), pp.161-170.

[9] Y.-C. Kao, C.-N. Lee, P.-J. Wu, and H.-H. Kao, "A Network Coding Equivalent Content Distribution Scheme for Efficient Peer-to-Peer Interactive VoD Streaming", Parallel and Distributed System, IEEE Transaction on, vol.23, no.6, (2012), pp.985-994.

[10] J. Krithiga and R.C. Porselvi, "Efficient CodeGuard mechanism against pollution attacks in interfow network coding", Communications and Signal Processing(ICCSP) 2014 International Conference on, (2014).

[11] C. Yao, P.-N. Chen, T.-Y. Wang and Y.S Han, "Performance Analysis and Code Design for Minimum Hamming Distance Fusion in Wireless Sensor Networks", Information Theory, IEEE Transactions on, vol.53, no.5, (2007), pp.1716-1734.

[12] $\mathrm{H}$. Wu and J. Zheng, "Efficient network coding-based multicast retransmission mechanism for mobile communication networks", Communications, IET, vol.6, no.2, (2012), pp.187-193.

[13] $\mathrm{H}$. Wu and J. Zheng, "network coding based multicast retransmission for mobile communication networks", Communications Technology (ICCT) 2012 12th IEEE International Conference on, (2010).

[14] $\mathrm{H}$. Wu and J. Zheng, CoRET, "A network coding based multicast retransmission scheme for mobile communication networks", in Proc. of IEEE International Conference on Communications (ICC'11), (2011).

[15] Y.C. Wang, S.R. Ye and Y.C. Tseng, "A fair scheduling algorithm with traffic classification in wireless networks", Computer Communications, vol.28, no.10, (2005), pp.1225-1239.

[16] Y. C. Wang, Y. C. Tseng, and W. T. Chen, "MR-FQ: a fair scheduling algorithm for wireless networks with variable transmission rates," Information Technology: Research and Education, 2005. ITRE 2005. 3rd International Conference on, (2005).

[17] K. Lu, Y. Qian, H.H. Chen and S. Fu, "WiMAX networks: from access to service platform", IEEE network, vol.22, no.3, (2008), pp.38-45.

[18] W.H Yang, Y.C Wang, Y.C Tseng and B.S.P Lin, "Energy efficient network selection with mobility 
pattern awareness in an integrated WiMAX and WiFi network", International Journal on Communication Systems, vol.23, no.2 (2010), pp.213-230.

[19] D. Nguyen, T. Nguyen and B. Bose, "Wireless Broadcasting Using Network Coding", Technical Report: OSU-TR-2006-06, Oregon State University, (2006).

[20] Q. Hu and J. Zheng, "An Efficient Packet Selection Algorithm for Network Coding Based Multicast Retransmission in Mobile Communication Networks", in Pro.of IEEE International Conference on Communication Technology(ICCT), (2011), NanJing,China.

[21] W.H. Yang, Y.C. Wang, and Y.C. Tseng, "Efficient packet recovery using prioritized network coding in DVB-IPDC systems”, IEEE Communications Letters, vol. 16, no.3, (2012), pp. 382-385.

\section{Authors}

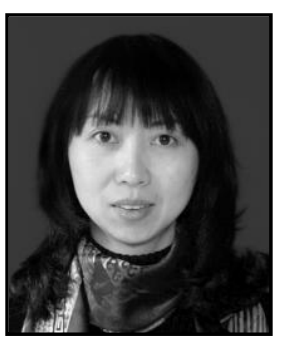

Lian Wang received the M.S. degree in computer and network engineering from London South University, London, UK, in 2004. And she is currently a Ph.D. candidate at Southwest Jiaotong University, Chengdu, China. She is an associate professor in the College of Computer Science and Technology, Chong Qing University of Posts and Telecommunications. Her research interests include network coding and wireless communication. Email: wanglian@cqupt.edu.cn

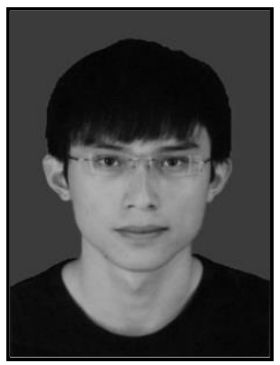

Jiehao Wang was born in Jiangsu Province, China, in 1989. He is current master students at Chongqing University of Posts and Telecommunications, His research interest is wireless network coding. Email: wjh123987@ hotmail.com 
International Journal of Future Generation Communication and Networking Vol. 8, No. 3 (2015) 